\title{
Gene expression profile analysis of the bone microenvironment in patients with spinal metastases
}

\author{
WANGMI LIU, YUN LIANG, CHONG BIAN, LIBO JIANG, GUOLI ZHENG and JIAN DONG \\ Department of Orthopedic Surgery, Zhongshan Hospital, Fudan University, Shanghai 200032, P.R. China
}

Received March 2, 2016; Accepted February 1, 2017

DOI: $10.3892 / \mathrm{ol} .2017 .7267$

\begin{abstract}
The present study aimed to identify the underlying molecular mechanisms associated with spinal metastases. Gene expression profiles in cancellous bone samples from the spines of five patients with spinal metastases, with different primary cancers, and three normal control patients were measured using microarray analysis and subsequently compared. The differentially expressed genes (DEGs) identified were filtered using bioinformatics analyses followed by cluster analysis, gene ontology (GO) term and Kyoto Encyclopedia of Genes and Genomes pathway enrichment analyses. Finally, a protein-protein interaction network was constructed and analyzed. A total of 152 upregulated and 388 downregulated DEGs were identified. The cluster analysis demonstrated a marked difference between the gene expression profiles of samples from patients with spinal metastases and those from normal patients. The GO terms enriched in the upregulated DEGs were associated with cell death, and those enriched in the downregulated DEGs were associated with the cell cycle. The upregulated DEGs were enriched in signaling pathways associated with tight junctions, and the downregulated DEGs were enriched in signaling pathways associated with porphyrin metabolism. In the PPI network constructed, transcription factor AP-1 and proliferating cell nuclear antigen had the highest connectivity degrees with the upregulated and downregulated DEGs, respectively. The gene expression profile data from the present study provides new insights into the underlying molecular mechanisms of spinal metastases, and will aid in the development of novel anticancer treatments.
\end{abstract}

\section{Introduction}

It is estimated that $>1.6$ million new cases of cancer were diagnosed in the United States of America in 2015 (1). Therefore,

Correspondence to: Professor Jian Dong, Department of Orthopedic Surgery, Zhongshan Hospital, Fudan University, 180 Fenglin Road, Xuhui, Shanghai 200032, P.R. China

E-mail: dong.jian@zs-hospital.sh.cn

Key words: spinal metastases, organ-specific metastases, differentially expressed genes, functional enrichment analysis, microarray cancer is one of the most common diseases and a major cause of mortality in modern society. Distant metastases, rather than cell proliferation itself, are lethal and contribute to $>90 \%$ of mortalities among patients with cancer (2). Although numerous breakthroughs in this field allow for the clinical management cancer, the underlying molecular mechanisms of metastasis remain poorly understood. Notably, numerous publications have reported that different types of cancer induce metastases preferentially at specific distal sites, which supports the concept of the 'seed and soil' theory proposed by Stephen Paget (3). According to this theory, the organ microenvironment serves a vital role in the formation of metastases. The organ in which metastasis from a certain type of cancer may or may not occur depends on the interaction between the disseminating cancer cells and the microenvironment of the organ itself.

Following the lungs and liver, bone is the third most common site of metastasis (4). According to data from autopsy studies, as many as 30-70\% of patients with cancer have spinal metastases (5), which demonstrates that the spine is a common site for skeletal metastases. Spinal metastases destroy the stability of the spine, leading to refractory pain, fractures and devastating neurologic consequences (6). This process results in a significant negative impact on morbidity and survival (7). Although patients have an increased number of therapeutic options because of improvements in multidisciplinary treatments, patients with spinal metastases still have a poor quality of life for the remaining course of their disease (8). This may be ascribed to the failure to appreciate that prevention, rather than the cure of spinal metastasis, may be a more successful approach. To achieve this, a comprehensive knowledge of the underlying molecular mechanisms of spinal metastasis is required.

To investigate the underlying molecular mechanisms of organ-specific metastasis, particularly in the bone and spine, researchers have examined the genes potentially involved in this process in different primary cancers, including breast (9), prostate $(10)$ and lung $(11,12)$ cancer. However, the majority of existing studies investigating spinal metastasis focus primarily on the analysis of the genetic alterations in the metastatic cells, rather than the microenvironment of the sites of metastasis in the spinal tissue. Given the interaction between disseminated cancer cells and the microenvironment of the spine, certain features of the gene expression of cancellous bone tissue in the spine may have changed to mediate and favor the colonization of cancer cells. Hence, investigating the gene expression 
profile of cancellous bone in spinal metastases may aid in future studies into the underlying molecular mechanisms of spinal metastasis.

In the present study, a microarray analysis was performed to identify differentially expressed genes (DEGs) in cancellous bone tissue from patients with spinal metastases compared with that from normal control patients. To explore different gene expression signatures and the underlying molecular mechanisms associated with spinal metastases, 5 patients with different primary cancers (lung, breast, liver, prostate and kidney cancer) were included. Additionally, gene ontology (GO) term and Kyoto Encyclopedia of Genes and Genomes (KEGG) pathway enrichment analyses were performed, and a protein-protein interaction (PPI) network was constructed to identify key (hub) genes. The current study aimed to provide a comprehensive perspective into the underlying molecular mechanisms, and the prevention and treatment, of spinal metastases.

\section{Materials and methods}

Patients and specimens. The present study was reviewed and approved by the Ethics Committee of Zhongshan Hospital (Fudan University, Shanghai, China). Written informed consent was obtained from all patients or their families. In total, 8 participants were enrolled, including 5 patients with spinal metastases (Group 1) and 3 normal controls (Group 2; Table I). Each of the 5 patients had a different primary cancer, including lung, breast, liver, prostate and kidney cancer, confirmed by pathological diagnosis. Between January 2012 and December 2015, each patient received a total en bloc spondylectomy, and cancellous bone tissue specimens $0.5 \mathrm{~mm}$ away from the metastatic spinal tumors were obtained and snap frozen in liquid nitrogen. The specimens were stored at $-80^{\circ} \mathrm{C}$ until gene expression analysis was performed. The 3 normal controls were patients who had undergone spinal surgery at Zhongshan Hospital due to non-cancerous diseases. The cancellous bone from their spines was collected via procedures that were already required during the surgery, such as decompression. These specimens were also stored at $-80^{\circ} \mathrm{C}$.

RNA isolation. Total RNA was extracted from each specimen using the RNeasy Protect Mini kit (Qiagen, Inc., Valencia, CA, USA) according to the manufacturer's protocol. The concentration of the RNA obtained was detected using a NanoDrop 1000 spectrophotometer (NanoDrop; Thermo Fisher Scientific, Inc., Wilmington, DE, USA). The RNA integrity was assessed via a Bioanalyzer 2100 (Agilent Technologies, Inc., Santa Clara, CA, USA). Specimens with an absorbance (A) 260/A280 ratio $>1.9$ and RNA integrity values $>8.0$ were used for further analysis.

Microarray analysis. Gene expression profiles were assessed using Illumina HumanHT-12_V4 BeadChip arrays (cat. no. 9479628056; Illumina Inc., San Diego, CA, USA). Each array contained $>47,000$ probes, including specific gene probes or probe sets derived from the National Center for Biotechnology Information RefSeq and UniGene databases (13). According to the manufacturer's protocol, reverse transcription to synthesize first strand complementary (c) DNA was primed with T7 Oligo (dT) Primer in order to synthesize cDNA containing a T7 promoter sequence. Single-stranded cDNA was subsequently converted into a double-stranded DNA, providing the template for transcription. During the amplification and labeling step, multiple copies of biotinylated cRNA from the double-stranded cDNA templates were generated. Following purification, the cRNA was ready for use with the Illumina direct hybridization array kits (14). The cRNA was hybridized to the bead arrays at $55^{\circ} \mathrm{C}$ for $18 \mathrm{~h}$ and then scanned using an Illumina iScan reader (cat. no. 9479628056; Illumina Inc., San Diego, CA, USA).

Data pre-processing, differential expression analysis and clustering. The initial array scan intensity data were analyzed using Illumina Genome Studio Gene Expression Module software (v1.1.1; Illumina Inc.). Data pre-processing, such as background adjustment, normalization and log transformation of the values, was performed. Furthermore, the probe-level data were converted to gene expression values. Where several probes corresponded to one gene, the mean value of the probe-level data was taken as the gene expression value. Cluster analysis was used to group the patients into clusters. Patients assigned to the same cluster are more closely related to one another compared with patients assigned to different clusters. An unpaired t-test analysis was used to identify the DEGs between the spinal metastasis and normal groups. Then, the $\log _{2}$ fold change value was calculated. The raw P-values were adjusted into false discovery rates (FDRs) using the Benjamin and Hochberg method as described previously (15). An FDR $<0.05$ and $\log _{2} \mathrm{FCl}>1$ were used as the cut-off criteria to identify significantly DEGs. Finally, the cluster analysis was used to group the cases into clusters according to the DEGs.

GO term and KEGG pathway enrichment analyses. GO (16) is a tool for the unification of biology in terms of biological processes, molecular functions and cellular components. KEGG (17) is a knowledge database used for classifying correlating gene sets into their respective signaling pathways. The Database for Annotation, Visualization and Integrated Discovery (DAVID) (18), a comprehensive set of functional annotation tools, is used for the systematic and integrative analysis of large gene lists. To analyze the DEGs at the functional level, GO term and KEGG pathway enrichment analyses were performed using the DAVID online tool to obtain the enriched GO terms and pathways via a clustering algorithm. $\mathrm{P}<0.05$ was set as the threshold value.

PPI network construction. The Search Tool for the Retrieval of Interacting Genes (STRING) database (19) is a pre-computed global resource, which was designed to explore and evaluate PPI information. In the present study, the PPI of the DEGs identified was screened with a required confidence (combined) score $>0.4$ using the STRING online tool (version 10.0). Then, the PPI network was constructed and visualized using Cytoscape (20), which is a general bioinformatics package to aid in visualizing biological networks and integrating PPI data. Given that the majority of the networks were scale-free, hub genes with a connectivity degree $>5$ were selected, as described previously (21). 
Table I. Clinical profiles of the patients with spinal metastasis and normal patients.

\begin{tabular}{lllll}
\hline Patient group & Sex & Age (years) & Primary disease & Location of specimen \\
\hline Group $1^{\text {a }}$ & & & & T4 \\
1 & Female & 75 & Breast cancer & T9 \\
2 & Female & 39 & Liver cancer & L2 \\
3 & Male & 50 & Prostate cancer & T10 \\
4 & Male & 57 & Kidney cancer & C4 \\
5 & Male & 80 & & C5 \\
Group 2b & Female & 65 & Spine fracture & L4 \\
1 & Male & 33 & Disc herniation & T6 \\
2 & Male & 63 & & \\
\hline
\end{tabular}

${ }^{\mathrm{a}}$ Group 1 represents the patients with spinal metastases; ${ }^{\mathrm{b}}$ Group 2 represents the normal controls. C, cervical spine; T, thoracic spine; L, lumbar spine.

\section{Results}

Identification of DEGs in spinal metastasis. The genes that were significantly upregulated or downregulated in the cancellous bone samples from patients with spinal metastases compared with the samples from normal patients were identified with a FDR $<0.05$ and a $\mid \log _{2} \mathrm{FCl}>1$. As a result, a total of 540 DEGs were obtained following data processing (data not shown). Among the DEGs, 152 were significantly upregulated and 388 were significantly downregulated.

Cluster analysis. The clustering of the DEGs demonstrated that the gene expression signature in samples from patients with spinal metastases more closely resembled each other compared with the normal controls (Fig. 1). There were notable differences between the cancellous bone from patients with spinal metastases and the normal controls according to their gene expression signatures.

GO term enrichment analysis. Based on GO term enrichment analysis using the DAVID tool, the DEGs were categorized into the following three major terms: Biological processes, molecular functions and cellular components. The top three GO terms of each of the categories are illustrated in Fig. 2. The enriched terms of the upregulated genes in samples from patients with spinal metastases were significantly associated with cell death, actin binding and cell-cell junctions in the three categories (Fig. 2A). The enriched terms identified among the downregulated genes in samples from patients with spinal metastases were significantly associated with the cell cycle, ATP binding and condensed chromosomes in the three categories (Fig. 2B).

KEGG pathway enrichment analysis. The DAVID tool was used to identify the KEGG biological pathways associated with the DEGs in the samples from patients with spinal metastases. The upregulated genes were significantly associated with tight junctions, adherence junctions and regulation of the actin cytoskeleton (Fig. 2C). By contrast, the downregulated genes from the spinal metastases were significantly associated

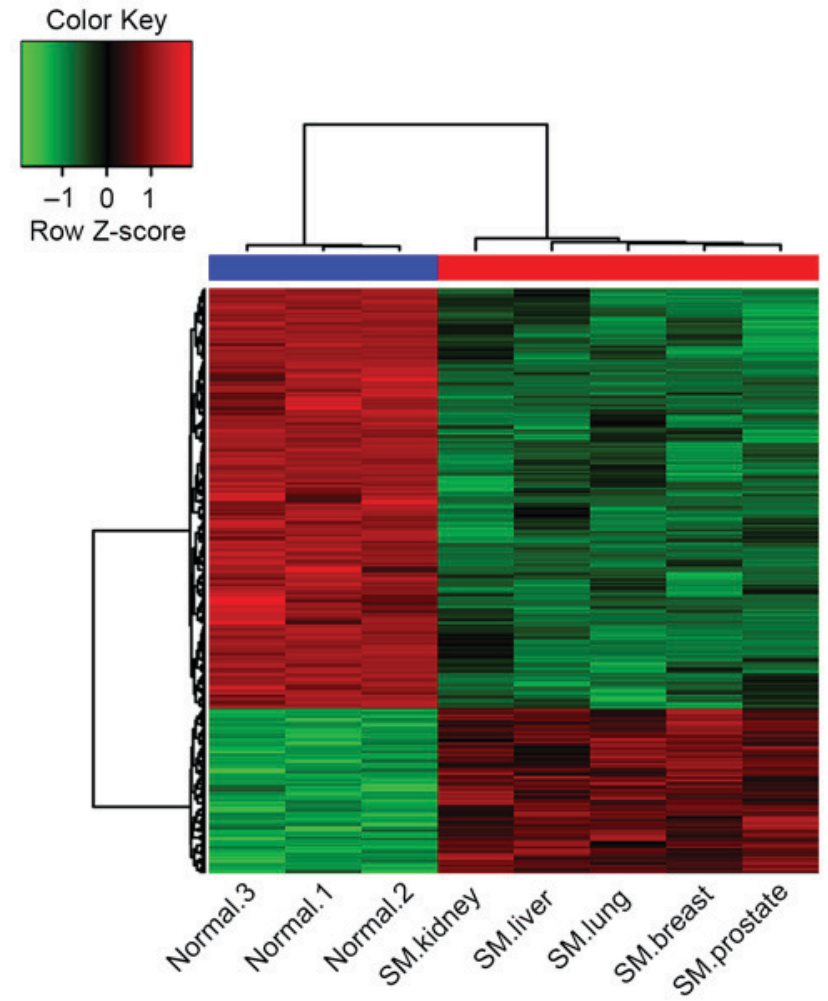

Figure 1. Heatmap of the DEGs in cancellous bone samples from normal patients and patients with spinal metastases. Red, upregulated DEGs; green, downregulated DEGs; SM, spinal metastases; DEGs, differentially expressed genes.

with porphyrin metabolism, the cell cycle and tumor protein p53 signaling pathway (Fig. 2C).

PPI network construction. Based on the STRING database, the PPI networks with hub genes possessing a connectivity degree $>5$ were constructed using Cytoscape. Networks with 51 and 30 nodes were obtained using the proteins encoded by the upregulated (Fig. 3A) and downregulated (Fig. 3B) genes, respectively. Within a PPI network, each node indicates 

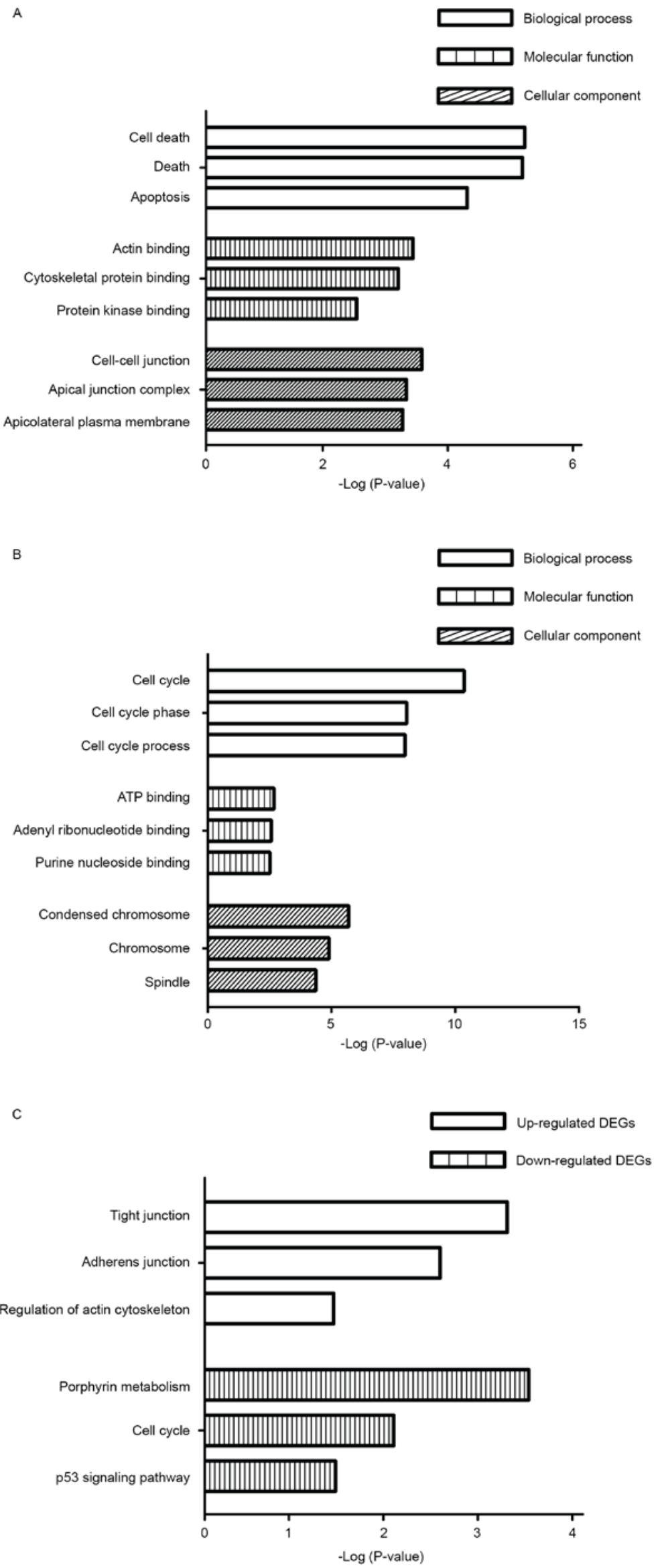

Figure 2. GO term and KEGG pathway enrichment analyses performed using DAVID on DEGs identified from cancellous bone samples from patients with spinal metastasis compared with samples from normal patients. (A) The enriched GO terms from the upregulated DEGs. (B) The enriched GO terms from the downregulated DEGs. (C) The enriched KEGG signaling pathways from the upregulated and downregulated DEGs. GO, gene ontology; KEGG, Kyoto Encyclopedia of Genes and Genomes; DEGs, differentially expressed genes. 


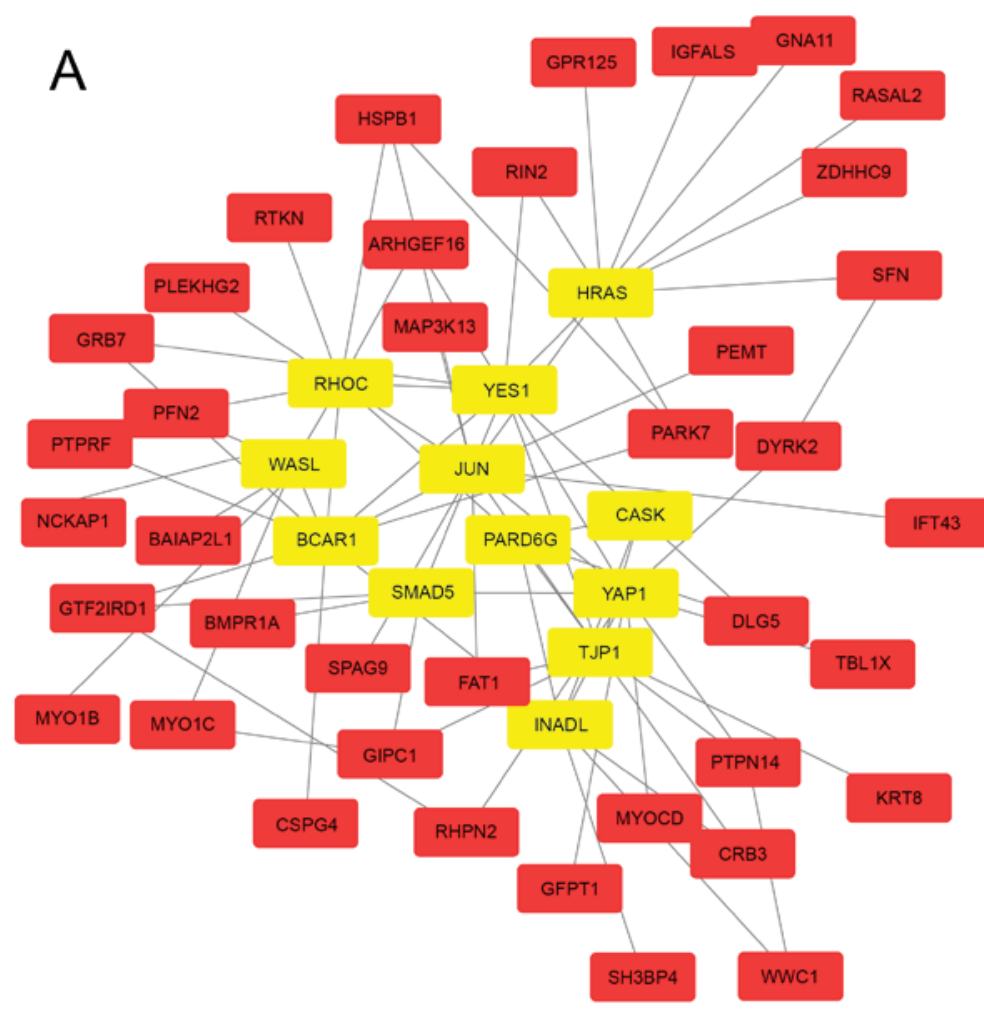

B

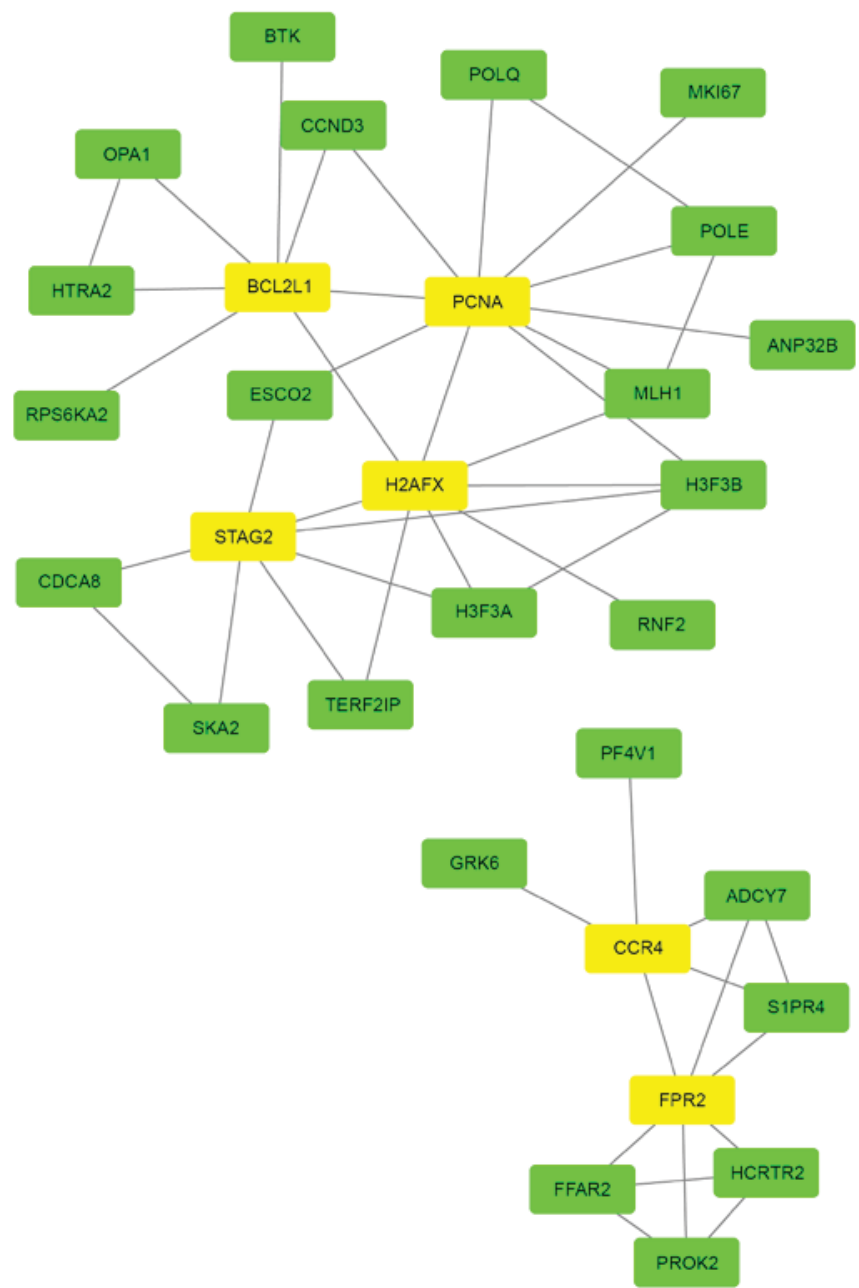

Figure 3. PPI network of the DEGs identified from cancellous bone samples from patients with spinal metastasis compared with samples from normal patients. (A) The PPI network of the upregulated DEGs. (B) The PPI network of the downregulated DEGs. Red, upregulated DEGs; green, downregulated DEGs; yellow, hub proteins. PPI, protein-protein interaction; DEGs, differentially expressed genes. 
a protein, and the lines between nodes indicate PPIs. The connectivity degree represents the number of lines linked to a given node, and nodes with a high connectivity degree $(\geq 5)$ are defined as hub genes that possess important biological functions. A total of 12 hub genes were selected from the upregulated PPI network, which included transcription factor AP-1 (JUN), GTPase HRas (HRAS) and Rho-related GTP-binding protein RhoC (RHOC) with connectivity degrees of 13, 10 and 10, respectively (Fig. 3A) Meanwhile, 6 hub genes, including proliferating cell nuclear antigen (PCNA), histone $\mathrm{H} 2 \mathrm{AX}$ (H2AFX) and cohesion subunit SA-2 (STAG), with connectivity degrees of 10,8 and 7, respectively, were identified from the downregulated PPI network (Fig. 3B).

\section{Discussion}

Although the advancement of surgical techniques has improved the quality of life of patients with spinal metastases (22), the underlying molecular mechanisms of this condition are not well understood. Therefore, studies investigating spinal metastases are required to develop effective prevention and therapy strategies. Recently, the bone marrow microenvironment has become an area of intense preclinical and clinical investigation. The bone microenvironment is composed of a mineralized extracellular matrix and specific cell types, which provide a unique and fertile 'soil' for cancer metastases. Cancer cells modify the bone microenvironment during their invasion and expansion by recruiting and modulating osteoclasts, osteoblasts, immune cells, vascular elements and bone matrix (23). Therefore, a better characterization of the interactions between cancer cells and the spinal microenvironment is essential for developments in this field.

In the present study, the microarray data generated from the cancellous bone tissue of 5 patients with spinal metastases and 3 normal patients was analyzed and 540 DEGs were identified. The different gene expression signatures demonstrate that the microenvironment of the bone marrow in spinal metastases is altered compared with the normal condition. The DEGs were subjected to an integrative systematic bioinformatics approach, including functional and pathway enrichment analyses, in addition to a PPI network construction. Based on these results, the underlying molecular mechanisms of spinal metastases could be explored at genetic and molecular levels, in order to provide further insights into spinal metastasis prevention and treatment.

The results of the cluster analysis, based on the DEGs, demonstrated marked differences between the cancellous bone from spinal metastases and that from normal patients. This result indicates that a change in the underlying gene activity is associated with spinal metastases. Notably, the gene expression signatures for breast cancer and prostate cancer were similar. In breast cancer, bone metastases are predominantly osteolytic (24), while bone metastases are predominantly osteoblastic in prostate cancer (25). They are thus theorized to represent two extremes of a continuum. However, data from the present study and previous research $(26,27)$ indicates that bone metastases typically have osteolytic and osteoblastic elements as a mixed phenotype. This may be ascribed to the interaction between osteoblastic and osteoclastic cells (28).
Functional enrichment analysis, based on GO, was performed in order to identify the underlying biological processes that the DEGs were associated with. In the present study, the enriched GO terms from the upregulated genes were primarily associated with cell death and actin binding in the cell-cell junctions. This may reflect the interaction between cancer cells and immune cells in spinal metastases, which indicates that tumor cells may acquire the ability to escape immune control or even eliminate immune cells, such as cluster of differentiation (CD) $4^{+}$and $\mathrm{CD}^{+} \mathrm{T}$ cells (29). The enriched GO terms from the downregulated genes were primarily associated with the cell cycle and ATP binding in condensed chromosomes. This suggests that immune cells persist around cancer cells in the bone marrow in a quiescent state (30). Therefore, cancer cells may escape immune surveillance via altering intrinsic tumor suppressor mechanisms in spinal metastases.

The pathway enrichment analysis based on KEGG evaluates differential expression patterns of gene groups rather than those of individual genes, and in cases in which the individual genes exhibit subtle biological function or property changes they are omitted by typical individual gene analysis (31). In the present study, the enriched pathways in the upregulated genes were predominantly associated with tight junctions, while the downregulated genes were associated with porphyrin metabolism. These results overlapped with the GO term enrichment analyses. Therefore, the data from the current study indicates an immunocompromised status in patients with spinal metastasis, which supports the findings from a previous study that a decline in the ability of the immune cells to recognize and destroy the tumor drives the dissemination of cancer cells to the bone (32).

A PPI network is necessary to understand the underlying molecular mechanisms of spinal metastasis, as the signal transduction network that responds to external and internal environmental stimuli is based upon interactions between proteins. The hub genes identified serve a vital role in this signal transduction network. The top hub genes identified in the present study were JUN and PCNA from the upregulated and downregulated genes, respectively. JUN interacts directly with specific target DNA sequences to regulate gene expression. For example, JUN participates in the receptor activator of nuclear factor kappa- $\beta$ (RANK)-RANK ligand (RANKL) system in osteolytic bone metastases. Briefly, signaling through RANK in osteoclast progenitors activates JUN, resulting in the differentiation of osteoclast progenitors into mature osteoclasts, which are responsible for bone resorption (33). Other hub genes, such as HRAS and RHOC, also act as signaling proteins with GTPase activity in activating osteoclasts (34). Thus, the activation of the RANK-RANKL system may serve a role in spinal metastases. PCNA is a cofactor of DNA polymerase $\delta$ and exists in the nucleus. PCNA acts as a homotrimer and increases the processivity of leading strand synthesis during DNA replication. Furthermore, in response to DNA damage, PCNA is ubiquitinated and is involved in the ubiquitin-conjugating enzyme E2 2-dependent DNA repair signaling pathway (35). Therefore, the relative lack of PCNA in the metastatic microenvironment could inhibit the maturation of immune cells while promoting the heterogeneity of cancer cells. H2AFX and STAG are also responsible for DNA 
replication, and their downregulation further exacerbates perturbations in the metastatic microenvironment.

The traditional view ascribes the frequency of spinal metastases to the specialized structure of the bone marrow in the spine. The first part of the structure is the vascular sinusoids that are lined with endothelial cells in the vertebra, have fenestrae of $60 \AA$ in diameter and lack a basement membrane (36). The second part is the marrow blood flow, which is relatively abundant in the vertebral bodies (37). However, the data from the present study revealed genetic changes in the metastatic microenvironment, which may be favorable to the metastasis, survival and growth of cancer cells in the spine, independent of the cancer type. This concept is in accordance with a previous study characterizing the importance of the interaction between cancer cells and the bone marrow in the vicinity of future metastatic sites (38). The extent to which DEGs in spinal metastasis are produced by the cancer cells themselves and by the microenvironment in response to the cancer cells requires further research.

There are a number of limitations to the present study. Firstly, the sample size for the microarray was small. Secondly, the results from the array and bioinformatics analysis lack corresponding in vitro experiments. Thus, genetic and experimental studies with a larger sample size are required to confirm the results from the current study.

In conclusion, based on the comprehensive set of bioinformatics analyses of microarray data, the results of the present study identified DEGs that are potentially associated with the molecular mechanisms of spinal metastasis in a number of cancer types. This will provide new insights into the underlying molecular mechanisms, prevention and treatment of spinal metastases. However, further experiments are required to confirm these results.

\section{Acknowledgements}

The present study was funded by the National Natural Science Foundation of China (grant no. 81572629).

\section{References}

1. Siegel RL, Miller KD and Jemal A: Cancer statistics, 2015. CA Cancer J Clin 65: 5-29, 2015.

2. Sporn MB: The war on cancer. Lancet 347: 1377-1381, 1996.

3. Paget $\mathrm{S}$ : The distribution of secondary growths in cancer of the breast. Cancer Metastasis Rev 8: 98-101, 1989.

4. Aaron AD: The management of cancer metastatic to bone. JAMA 272: 1206-1209, 1994.

5. Jacobs WB and Perrin RG: Evaluation and treatment of spinal metastases: An overview. Neurosurg Focus 11: e10, 2001.

6. Sciubba DM,Petteys RJ, Dekutoski MB, Fisher CG, Fehlings MG, Ondra SL, Rhines LD and Gokaslan ZL: Diagnosis and management of metastatic spine disease. A review. J Neurosurg Spine 13: 94-108, 2010

7. Tokuhashi Y, Matsuzaki H, Oda H, Oshima M and Ryu J: A revised scoring system for preoperative evaluation of metastatic spine tumor prognosis. Spine (Phila Pa 1976) 30: 2186-2191, 2005.

8. Ibrahim A, Crockard A, Antonietti P, Boriani S, Bünger C, Gasbarrini A, Grejs A, Harms J, Kawahara N, Mazel C, et al: Does spinal surgery improve the quality of life for those with extradural (spinal) osseous metastases? An international multicenter prospective observational study of 223 patients. Invited submission from the Joint Section Meeting on Disorders of the Spine and Peripheral Nerves, March 2007. J Neurosurg Spine 8: 271-278, 2008
9. Kang Y, Siegel PM, Shu W, Drobnjak M, Kakonen SM, Cordón-Cardo C, Guise TA and Massagué J: A multigenic program mediating breast cancer metastasis to bone. Cancer Cell 3: 537-549, 2003.

10. Jamieson WL, Shimizu S, D'Ambrosio JA, Meucci O and Fatatis A: CX3CR1 is expressed by prostate epithelial cells and androgens regulate the levels of CX3CL1/fractalkine in the bone marrow: Potential role in prostate cancer bone tropism. Cancer Res 68: 1715-1722, 2008.

11. Cai X, Luo J, Yang X, Deng H, Zhang J, Li S, Wei H, Yang C, $\mathrm{Xu} \mathrm{L}$, Jin $\mathrm{R}$, et al: In vivo selection for spine-derived highly metastatic lung cancer cells is associated with increased migration, inflammation and decreased adhesion. Oncotarget 6: 22905-22917, 2015.

12. Dat le T, Matsuo T, Yoshimaru T, Kakiuchi S, Goto H, Hanibuchi M, Kuramoto T, Nishioka Y, Sone S and Katagiri T: Identification of genes potentially involved in bone metastasis by genome-wide gene expression profile analysis of non-small cell lung cancer in mice. Int J Oncol 40: 1455-1469, 2012.

13. Wheeler DL, Church DM, Federhen S, Lash AE, Madden TL, Pontius JU, Schuler GD, Schriml LM, Sequeira E, Tatusova TA and Wagner L: Database resources of the National Center for Biotechnology. Nucleic Acids Res 31: 28-33, 2003.

14. Wozniak MB, Le Calvez-Kelm F, Abedi-Ardekani B, Byrnes G, Durand G, Carreira C, Michelon J, Janout V, Holcatova I, Foretova $\mathrm{L}$, et al: Integrative genome-wide gene expression profiling of clear cell renal cell carcinoma in Czech Republic and in the United States. PLoS One 8: e57886, 2013.

15. Reiner A, Yekutieli D and Benjamini Y: Identifying differentially expressed genes using false discovery rate controlling procedures. Bioinformatics 19: 368-375, 2003.

16. Ashburner M, Ball CA, Blake JA, Botstein D, Butler H, Cherry JM, Davis AP, Dolinski K, Dwight SS, Eppig JT, et al: Gene ontology: Tool for the unification of biology. The Gene Ontology Consortium. Nat Genet 25: 25-29, 2000.

17. Kanehisa M and Goto S: KEGG: Kyoto encyclopedia of genes and genomes. Nucleic Acids Res 28: 27-30, 2000.

18. Huang da W, Sherman BT and Lempicki RA: Systematic and integrative analysis of large gene lists using DAVID bioinformatics resources. Nat Protoc 4: 44-57, 2009.

19. von Mering C, Huynen M, Jaeggi D, Schmidt S, Bork P and Snel B: STRING: A database of predicted functional associations between proteins. Nucleic Acids Res 31: 258-261, 2003.

20. Smoot ME, Ono K, Ruscheinski J, Wang PL and Ideker T: Cytoscape 2.8: New features for data integration and network visualization. Bioinformatics 27: 431-432, 2011.

21. Kou Y, Zhang S, Chen X and Hu S: Gene expression profile analysis of colorectal cancer to investigate potential mechanisms using bioinformatics. Onco Targets Ther 8: 745-752, 2015.

22. Wu J, Zheng W, Xiao JR, Sun X, Liu WZ and Guo Q: Health-related quality of life in patients with spinal metastases treated with or without spinal surgery: A prospective, longitudinal study. Cancer 116: 3875-3882, 2010.

23. Weilbaecher KN, Guise TA and McCauley LK: Cancer to bone: A fatal attraction. Nat Rev Cancer 11: 411-425, 2011.

24. Coleman RE and Seaman JJ: The role of zoledronic acid in cancer: Clinical studies in the treatment and prevention of bone metastases. Semin Oncol 28 (2 Suppl 6): S11-S16, 2001.

25. Charhon SA, Chapuy MC, Delvin EE, Valentin-Opran A, Edouard CM and Meunier PJ: Histomorphometric analysis of sclerotic bone metastases from prostatic carcinoma special reference to osteomalacia. Cancer 51: 918-924, 1983.

26. Guise TA: The vicious cycle of bone metastases. J Musculoskelet Neuronal Interact 2: 570-572, 2002.

27. Kraljevic Pavelic S, Sedic M, Bosnjak H, Spaventi S and Pavelic K: Metastasis: New perspectives on an old problem. Mol Cancer 10: 22, 2011.

28. Ren G, Esposito M and Kang Y: Bone metastasis and the metastatic niche. J Mol Med (Berl) 93: 1203-1212, 2015.

29. Zhang K, Kim S, Cremasco V, Hirbe AC, Collins L, Piwnica-Worms D, Novack DV, Weilbaecher K and Faccio R: $\mathrm{CD} 8+\mathrm{T}$ cells regulate bone tumor burden independent of osteoclast resorption. Cancer Res 71: 4799-4808, 2011.

30. Schreiber RD, Old LJ and Smyth MJ: Cancer immunoediting: Integrating immunity's roles in cancer suppression and promotion. Science 331: 1565-1570, 2011.

31. Nam D and Kim SY: Gene-set approach for expression pattern analysis. Brief Bioinform 9: 189-197, 2008.

32. Capietto AH and Faccio R: Immune regulation of bone metastasis. Bonekey Rep 3: 600, 2014. 
33. Mundy GR: Metastasis to bone: Causes, consequences and therapeutic opportunities. Nat Rev Cancer 2: 584-593, 2002.

34. Russell RG: Bisphosphonates: The first 40 years. Bone 49: 2-19, 2011.

35. Hoege C, Pfander B, Moldovan GL, Pyrowolakis G and Jentsch S: RAD6-dependent DNA repair is linked to modification of PCNA by ubiquitin and SUMO. Nature 419: 135-141, 2002.

36. De Bruyn PP: Structural substrates of bone marrow function. Semin Hematol 18: 179-193, 1981.

37. Kahn D, Weiner GJ, Ben-Haim S, Ponto LL, Madsen MT, Bushnell DL, Watkins GL, Argenyi EA and Hichwa RD: Positron emission tomographic measurement of bone marrow blood flow to the pelvis and lumbar vertebrae in young normal adults. Blood 83: 958-963, 1994.
38. Kaplan RN, Psaila B and Lyden D: Bone marrow cells in the 'pre-metastatic niche': within bone and beyond. Cancer Metastasis Rev 25: 521-529, 2006. International (CC BY-NC-ND 4.0) License. 